\title{
Maximal intermittent handgrip strategy: design and evaluation of an exercise protocol and a grip tool
}

This article was published in the following Dove Press journal:

Clinical Interventions in Aging

10 May 2016

Number of times this article has been viewed

\section{Danielle Christine Bentley Scott Gordon Thomas}

Faculty of Kinesiology and Physical Education, University of Toronto, Toronto, Ontario, Canada

Correspondence: Danielle Christine Bentley

Faculty of Kinesiology and Physical Education, University of Toronto, 55 Harbord Street, Toronto, ON, Canada M5S 2W6

Tel + I 4169465637

Email danielle.bentley@mail.utoronto.ca
Abstract: Handgrip (HG) exercise has been prescribed as a lifestyle intervention to successfully reduce resting blood pressure (BP) among heterogeneous groups of participants. Current HG protocols have limited accessibility due to complicated exercise prescriptions and sophisticated required equipment. Therefore, this research describes the design and evaluation of the maximal intermittent (MINT) HG exercise strategy, consisting of both a novel exercise protocol ( $32 \times 5$ seconds maximal grip squeezes separated by 5 seconds of rest between sets) and an original grip tool. This research was a multistep progressive design that included 51 postmenopausal women as participants in three separate research studies. Part 1 of this research focuses on the MINT exercise protocol. A literature-informed rationale for the design of the protocol is described. This includes exercise intensity, work-to-rest ratio, and total exercise duration with reference to the unique physiology (mechanoreflex and metaboreflex) of postmenopausal women. Subsequent experimental analyses of acute responses to the MINT protocol revealed that women produced $50 \%$ of their maximum grip force with moderate cardiovascular responses (increases of systolic BP: $41.6 \mathrm{mmHg}$, diastolic BP: $20.1 \mathrm{mmHg}$, heart rate: $35.1 \mathrm{bpm}$ ) that remained far below the thresholds of concern identified by the American College of Sports Medicine. Part 2 of this research describes the creation of a novel grip tool, beginning with a mixed-methods assessment of participant opinions regarding two distinct in-laboratory grip tools, leading to the creation of four prototype MINT tools. Structured focus groups revealed a strong preference for MINT prototype 1 for all tool design features, including color, shape, size, and foam grip. Collectively, the result of this multistep research is a novel HG exercise strategy with enhanced accessibility by being easy to understand and simple to execute. The long-term training effectiveness of MINT as an exercise intervention for the reduction of resting BP has yet to be determined.

Keywords: handgrip exercise, blood pressure, new product development, novel exercise strategy, postmenopausal women, exercise safety

\section{Introduction}

Recently, there has been a strong expression of desire by both clinicians and patients for research dedicated toward alternative treatment options, such as lifestyle interventions, for the management and improvement of health. ${ }^{1}$ As a lifestyle intervention, physical activity and exercise can improve health through the management of resting blood pressure (BP). ${ }^{2}$ Specifically, isometric handgrip (HG) exercise has been prescribed as a lifestyle intervention to successfully reduce resting BP among heterogeneous groups of participants. ${ }^{3-6}$ Within this literature, the most common HG training protocols prescribe sustained grip squeezes, typically $4 \times 2$ minutes contractions, at low to moderate intensities of $20 \%-50 \%$ maximal volitional contraction (MVC), with 1-5 minutes of rest between sets, performed three to five times per week for a total of 4-10 weeks. ${ }^{4,5}$ With a relatively small time commitment ( $<20$ minutes/session) 
and substantial BP reductions (mean [CI]) of systolic BP (SBP; $-6.9 \mathrm{mmHg}[-8.4 \mathrm{mmHg}$ to $-5.6 \mathrm{mmHg}]$ ) and diastolic BP (DBP; $-3.6 \mathrm{mmHg}[-4.8 \mathrm{mmHg}$ to $-2.6 \mathrm{mmHg}]),{ }^{6}$ HG exercise has the potential to be an impactful lifestyle intervention.

Resting BP reduction is especially important for cohorts at an increased risk of cardiovascular disease (CVD), such as postmenopausal women. Following the menopause transition women experience various physiological changes including loss of muscular strength and balance, ${ }^{7}$ and an exponential rise in CVD incidence. ${ }^{8}$ Despite compelling scientific research and widespread public health recommendations, among women 45-64 years and 65-74 years old only $18 \%$ and $11 \%$, respectively, perform physical activities that enhance and maintain muscle strength and endurance two or more times per week. ${ }^{9}$ An accessible and effective exercise option, such as HG exercise, may improve postmenopausal women's health through BP reduction while also introducing positive exercise behaviors in this at-risk population.

Currently, the accessibility of HG exercise is limited by the use of MVC percentages to prescribe intensity, as this requires that force output during each exercise bout be monitored via supervised laboratory visits and/or specialized at-home exercise equipment. Attempts to improve the accessibility of HG exercise while preserving experimental control of moderate-intensity force prescription have used less sophisticated grip tools with resistance-specific rubber bands ${ }^{10}$ and springs. ${ }^{11,12}$ However, such tools still require regular laboratory visits to assess training-induced changes in grip strength and consequently adjust the spring and/or band tension throughout the training program. To eliminate the necessity for either specialized equipment or frequent laboratory follow-ups, HG exercise can be prescribed as maximal grip force.

Protocols using maximal grip force against generic grip objects have successfully induced positive CV adaptations such as collateral vascular recruitment and growth, ${ }^{13}$ reduced BP and improved local blood flow, ${ }^{14}$ and reduced ergoreflex activity. ${ }^{15}$ Despite these positive impacts on $\mathrm{CV}$ health indices, recommendation of this accessible exercise option by health care providers and exercise professionals is rare. Experimental evidence documenting the safety of maximal HG exercise by examining the acute physiological responses may facilitate acceptance of maximal grip exercise for athome training. Furthermore, the HG literature currently lacks a comprehensive assessment of participant perceptions and opinions of HG exercise. Together, this information would supplement the growing body of literature in support of HG exercise training.

\section{General research outline}

This manuscript describes the design and evaluation of the maximal intermittent (MINT) HG exercise strategy, consisting of both a novel exercise protocol (Part 1) and an original grip tool (Part 2). Part 1 begins with a literature-informed rationale for the design of a highly accessible $\mathrm{HG}$ exercise protocol that specifically considers the unique physiological responses of postmenopausal women to HG exercise. Subsequent experimental analyses of this protocol quantify grip performance while following the maximal intensity prescription, describe the acute CV responses to MINT in reference to published exercise safety recommendations, and collect participant feedback concerning the MINT protocol. Part 2 describes the development of a participantinformed grip tool, beginning with a mixed-methods assessment of feedback to two distinct in-laboratory grip tools. These results informed the creation of four mock-MINT tools for critique and comparison using structured focus groups, with participant-preferred design features resulting in the final MINT grip tool. Throughout this manuscript, MINT is compared to ZONA (ZonaPLUS; Zona Health, Boise, ID, USA), a commonly used commercial device with a preset exercise protocol of $4 \times 2$ minutes sustained grip contractions at $30 \%$ MVC with 1 minute of rest in between sets.

All components of the research were approved by the Research Ethics Board at the University of Toronto (REB\#29450, REB\#31450). Experimental procedures are in accordance with the guidelines set forth by the World Medical Association in the Declaration of Helsinki. ${ }^{16}$ At each stage of this research, participants received a full explanation of experimental procedures, exercise protocols, and possible risks associated with participation in the study prior to providing written informed consent. Testing was performed in the Human Health and Performance Laboratory at the University of Toronto.

\section{Part I: the MINT exercise protocol Protocol design rationale}

The primary goal in designing the MINT HG exercise protocol was to enhance accessibility by being easy to understand and simple to execute. Conceptualization of MINT addressed three distinct $\mathrm{HG}$ protocol design features: 1) exercise intensity, 2) work-rest timing cycles, and 3) total exercise duration. 
Directly aligned with the primary design goal, maximal grip force was selected as the MINT exercise intensity due to its ease and simplicity. In addition, maximal grip exercise self-calibrates over time to the individual's changing grip strength, ideal for prolonged periods of at-home training.

Previous literature using maximal grip intensity exercise for at-home training has included heterogeneous and inconsistent $\mathrm{HG}$ protocols with work-rest timing cycles of 1:1 second, ${ }^{14} 10: 20$ seconds,${ }^{15} 40$ grips $/ \mathrm{min},{ }^{15}$ and $1: 1$ minute, ${ }^{13}$ and total exercise durations of 30 minutes,${ }^{14} 10$ minutes,${ }^{15}$ and 40 minutes. ${ }^{13}$ None of the previous research has experimentally quantified actual grip performance in reference to the prescribed maximal intensity, an important consideration when evaluating the acute $\mathrm{CV}$ responses. Nonetheless, in response to the aforementioned maximal protocols, moderate increases in SBP have been reported (rest \pm standard deviation [SD] to peak $\pm \mathrm{SD}$ ) among participants with chronic heart failure (128.1 $\pm 4.9 \mathrm{mmHg}$ to $176.0 \pm 25.3 \mathrm{mmHg}),{ }^{15}$ coronary artery disease $(135.5 \pm 12.8 \mathrm{mmHg}$ to $142.6 \pm 11.8 \mathrm{mmHg}),{ }^{13}$ and healthy controls $(132.3 \pm 5.1 \mathrm{mmHg}$ to $189.6 \pm 20.9 \mathrm{mmHg}){ }^{15}$ Acute physiological alterations brought on by an exercise bout are correlated to long-term adaptations elicited by that training. ${ }^{17}$ Long-term HG training-induced reductions in resting $\mathrm{BP}$ are postulated to result from improvements in autonomic nervous control of neurocardiac function and vascular structures and/or structural changes to the vasculature in response to perturbations to local forearm blood flow. ${ }^{4,5}$ Therefore, these were the regulatory factors of focus when selecting the MINT work-rest timing cycle and the MINT total exercise duration. Where possible, the physiological response patterns of older women were specifically considered.

During an acute exercise bout, the autonomic nervous system acutely alters BP and heart rate (HR) via central command and the exercise pressor reflex (EPR). Central command is a feedforward neural mechanism that increases BP through both reductions of parasympathetic nerve activity and increases of sympathetic nerve activity. ${ }^{18-20}$ The influence of central command in driving the acute $\mathrm{CV}$ response to exercise can be estimated by examining the correlation between an individual's rating of perceived exercise exertion and their corresponding CV responses. ${ }^{21}$ In contrast, EPR provides feedback from muscle mechanoreceptors (mechanoreflex: group III fibers) and metaboreceptors (metaboreflex: group IV fibers). ${ }^{22}$ The mechanoreflex is rapidly stimulated by the physical manipulation of intramuscular receptors, proportional to working muscle tension. ${ }^{23-25}$ Therefore, the influence of the mechanoreflex in driving acute $\mathrm{CV}$ responses to exercise can be estimated by examining the correlation between an individual's exercise intensity output and their corresponding CV responses. ${ }^{23-25}$ During prolonged static contractions, the discharge rate of group III fibers decreases as the working muscle fatigues, ${ }^{23,24}$ while the metaboreflex is stimulated by exercise metabolites such as $[\mathrm{H}+],[\mathrm{K}+]$, bradykinin, lactic acid, and adenosine. ${ }^{22,26}$ Metabolite accumulation is amplified with prolonged static contractions at intensities ( $>20 \%$ MVCs) that limit local blood flow to the working skeletal muscle. ${ }^{27-29}$

The design of the MINT exercise protocol considered these three neural regulatory pathways with focus on the responsiveness of these pathways among older women. Recent research has identified that older women have an overactive metaboreflex that is associated with enhanced peripheral vasoconstriction ${ }^{30}$ and an overactive mechanoreflex. ${ }^{31}$ Therefore, the MINT protocol was designed to minimize the influence of each branch of the EPR during the HG exercise bout. To minimize the mechanoreflex time in grip contraction was limited to 5 seconds, as opposed to previous durations of 10 seconds ${ }^{15}$ and 1 minute. ${ }^{13}$ The metaboreflex was limited by integrating equal intervals of rest to reduce impedance of blood flow and facilitate hyperemic flow, providing adequate oxygenated blood and removing metabolic by-products. ${ }^{24}$ Young men and women completing a 5 seconds -5 seconds work-rest timing cycle have produced high-intensity grip ( $50 \% \mathrm{MVCs})$ for $>13$ minutes with high tolerability. ${ }^{32}$

With a maximal HG exercise intensity and a work-rest cycle of 5 seconds -5 seconds, the final protocol design feature to be selected was the total exercise duration. In line with the primary design goal of high accessibility, a shorter exercise duration was preferred while attempting to align the force-time integral of impulse between MINT and the preset ZONA protocol. Previous research using the ZONA automated digital grip device has reported that during an individual $\mathrm{HG}$ exercise bout, participants are able to achieve high exercise compliance $\left(97 \%,{ }^{33} 100 \%,{ }^{34} 95 \%\right.$, unpublished in-laboratory work). Using a similar pattern of compliance to MINT, total exercise time was

$$
\begin{aligned}
& (\text { ZONA force }) \times(\text { ZONA time }) \\
& \quad=(\text { MINT force }) \times(\text { MINT time }) \\
& (30 \% \text { of maximum force }) \times(480 \mathrm{~s}) \\
& \quad=(90 \% \text { of maximum force }) \times(\text { MINT time }) \\
& \frac{144(\% \mathrm{~N} \cdot \mathrm{s})}{0.9(\% \mathrm{~N})}=(\text { MINT time }) \\
& 160 \text { seconds }=\text { MINT time }
\end{aligned}
$$


The result of this sequential design process was the MINT HG exercise protocol, consisting of $32 \times 5$ seconds maximal grip squeezes separated by 5 seconds of rest inbetween sets.

The MINT protocol was then experimentally analyzed to quantify grip performance, determine acute $\mathrm{CV}$ responses, and collect participant feedback.

\section{Experimental analyses}

The experimental analyses of the MINT exercise protocol occurred as two sequential investigations. First, maximal HG exercise was completed by postmenopausal, healthy, normotensive (SBP $<120 \mathrm{mmHg}$ ) women (NT women) as part of a larger physiological comparison study (Bentley and Thomas 2015, unpublished data). After which, a second cohort of postmenopausal women with above-optimal resting $\mathrm{BP}$ and various comorbidities and medications (AO women) was recruited to confirm previous findings.

\section{Purpose}

The experimental purpose was to 1) quantify grip force performance while completing the MINT protocol, 2) compare the acute $\mathrm{CV}$ responses to maximal grip exercise to published exercise safety recommendations, and 3) collect participant feedback regarding the MINT protocol.

\section{Methodology}

The experimental procedures were similar for both the NT and the $\mathrm{AO}$ cohorts and took place following both a familiarization visit (to acclimatize participants to the laboratory assessments) and a baseline visit (for baseline recordings of maximum grip strength [1RM]). All experimental visits were conducted between 2 and $8 \mathrm{pm}$ to limit the impact of diurnal variations in $\mathrm{BP}^{35}$ with participants completing a 4-hour fast, 4-hour abstinence from caffeine, and a 24-hour abstinence from both alcohol and strenuous activity.

Participants arrived at the laboratory, completed the state-trait anxiety inventory ${ }^{36}$ (to examine the influence of stress on BP), and completed 15 minutes of seated and supported rest. The left arm was supported and fitted with an automated oscillometric brachial cuff (BpTRU Vital Signs made by BpTRU Medical Devices, model BPM-100, $\mathrm{BC}$, Canada) for discontinuous BP and HR recordings during both the resting and the postexercise recovery time points. The right arm was supported at heart level and fitted with a photoplethysmographic finger cuff (Finometer MIDI Model 2; Finapres Medical Systems, Amsterdam, the Netherlands) on the middle phalanx of the right third digit for beat-to-beat recordings of BP and HR both during rest and throughout each exercise bout. Recordings from the final 10 minutes of seated rest from each device were used to calculate day-of resting values. The BpTRU was then turned off while the brachial cuff remained in position on the left arm. Without changing position, participants completed the MINT exercise protocol against a grip dynamometer (ADInstruments Inc., Colorado Springs, CO, USA) using their nondominant hand (left for all), while Finometer continued to record the acute during-exercise responses of BP and HR. A timed PowerPoint (Microsoft Office Version 7.0, 2010) presentation guided participants through the prescribed protocol timing of $32 \times 5$ seconds maximal grip contractions with 5 seconds of rest in between sets. Participants verbally indicated their rating of perceived exertion (RPE: 6-20 scale $^{37}$ ) at the midway point and at the end of the exercise bout. Following exercise cessation, participants in the initial cohort only (healthy normotensive women) remained in the seated position for 30 minutes of recovery with $\mathrm{BP}$ and $\mathrm{HR}$ recorded (BpTRU) every 3 minutes.

To quantify grip force performance while completing MINT, real-time force data collected throughout the exercise bout (LabChart, 2010) were calculated as 1-second averages and converted to intensity percentages relative to an individual's 1RM (1RM determined as the maximum 1-second force output from three consecutive 5-second grips during the baseline visit). Grip performance is expressed as the average performance throughout the working time and the logarithmic decline in force over time. The during-exercise $\mathrm{CV}$ responses of SBP, DBP, and HR were collected as beatto-beat changes from day-of rest (Finometer) and have been expressed as both an average change value (Avg) and a peak 5-second change value (Peak). Statistical correlations were completed to indirectly determine the potential influence of central command and mechanoreflex in driving the acute $\mathrm{CV}$ response to MINT exercise. To assess participant feedback of MINT, RPEs were acquired at the midway point and at the end of the MINT exercise bout. Additional feedback collected from participants differed between participant groups. The initial NT cohort completed exit surveys to rate (1-10 scale) their enjoyment of the exercise protocol, the ease of the protocol, and if the protocol was fatiguing. Net promoter scores were also calculated using the answer to a single question, with a $0-10$ scale: How likely is it that you would recommend [MINT] to a friend or colleague? Respondents were grouped into promoters (9-10), passives 
(7-8), and detractors (0-6). Subtracting the percentage of detractors from the percentage of promoters yields the net promoter score, which can range from a low of -100 (if every customer is a detractor) to a high of 100 (if every customer is a promoter). ${ }^{38}$ The subsequent cohort of $\mathrm{AO}$ women provided open-ended feedback regarding the exercise protocol at the end of the experimental visit. Participant feedback regarding the MINT protocol was compared (dependent $t$-test) to feedback regarding the ZONA protocol (acquired during an experimental visit on another day) to determine relative acceptability. All data are presented as mean \pm SD with statistical significance set at $P<0.05$.

\section{Results}

The initial cohort (NT women) included 20 older (57.7 \pm 5.2 years), postmenopausal (8.4 \pm 5.6 years) women with optimal resting CV measures (SBP: 109.1 $\pm 9.1 \mathrm{mmHg}$, DBP: $73.3 \pm 7.7 \mathrm{mmHg}$, HR: $71.5 \pm 7.0 \mathrm{bpm}$ ) who self-reported to be active (Rapid Assessment of Physical Activity [RAPA]: $7.4 \pm 2.1$ ) and free from comorbidities (Bentley and Thomas 2015, unpublished data). The subsequent cohort (AO women) included eleven older ( $62.5 \pm 3.9$ years), postmenopausal (8.1 \pm 5.1 years) women who self-reported to be moderately active (RAPA: 6.0 \pm 2.4 ), with various comorbidities, including prehypertension ( $\mathrm{n}=7$, two on BP medication), hypertension ( $n=4$, two on BP medication), arthritis ( $n=2)$, Raynaud's disease $(n=1)$, diabetes $(n=1)$, osteoporosis $(n=1)$, asthma $(n=2)$, arterial fibromuscular dysplasia $(n=1)$, and palmar fibromatosis $(n=1)$. All AO women presented with aboveoptimal resting $\mathrm{CV}$ measures (SBP: $134.3 \pm 12.5 \mathrm{mmHg}$, DBP: $80.6 \pm 9.4 \mathrm{mmHg}$, HR: $73.7 \pm 8.0 \mathrm{bpm}$ ).

All participants successfully completed the MINT HG exercise protocol without experiencing clinical abnormalities or inappropriate symptoms during or immediately after exercise. As is typical during $\mathrm{HG}$ exercise, some $(\mathrm{n}=4)$ participants experienced mild-to-moderate local muscular fatigue. The initial cohort of NT women had an average grip performance while exerting maximal effort of $50.4 \%$, with the change in force over time characterized as a natural logarithmic decline $\left(y=-6.4 \ln (x)+80.92, R^{2}=0.59\right)$ (Figure 1A). Similar grip force performance was achieved by the subsequent cohort of AO women (average force: $49.7 \%$, logarithmic decline: $\left(y=-7.3 \ln (x)+84.2, R^{2}=0.64\right)$ (Figure 1B).

Among NT women, the MINT exercise protocol caused elevations in SBP (Peak: 42.2 $\pm 14.3 \mathrm{mmHg}$ ), DBP (Peak: 21.2 $\pm 8.1 \mathrm{mmHg}$ ), and HR (Peak: 13.8 $\pm 7.1 \mathrm{mmHg}$ ), all $P<0.05$. Following cessation of exercise, all $\mathrm{CV}$ variables returned to day-of resting values within the first recovery window (0-15 minutes) and remained at rest for the 30-minute postexercise duration (Figure 2). Among the AO women, MINT exercise elicited elevations in SBP only (Peak: $40.9 \pm 18.2 \mathrm{mmHg}, P<0.05)$, as the Peak acute responses of DBP (Peak: 18.9 $\pm 16.5 \mathrm{mmHg}, P>0.05$ ) and HR (Peak: $21.3 \pm 15.0 \mathrm{mmHg}, P>0.05)$ were in significant (Figure 3).

Examination of the average during-exercise CV responses reveals an interesting comparison between cohorts. Although the absolute average SBP value throughout MINT was greater in the AO cohort (NT: 132.2 $\pm 4.0 \mathrm{mmHg}$, AO: $157.1 \pm 6.0 \mathrm{mmHg}, P<0.05$ ), this difference can be attributed to an augmented resting SBP since there was no difference in change scores between the cohorts (NT: $24.1 \pm 2.7 \mathrm{mmHg}, \mathrm{AO}: 23.2 \pm 5.9 \mathrm{mmHg}$, $P>0.05)$. In comparison, the absolute average DBP values were similar between cohorts (NT: $83.5 \pm 2.0 \mathrm{mmHg}, \mathrm{AO}$ : $84.3 \pm 5.6 \mathrm{mmHg}, P>0.05)$ despite different resting values (NT: $72.6 \pm 1.9 \mathrm{mmHg}$, AO: $80.6 \pm 2.8 \mathrm{mmHg}, P<0.05$ ), due to trending change scores (NT: $10.9 \pm 1.5 \mathrm{mmHg}, \mathrm{AO}$ : $3.9 \pm 4.7 \mathrm{mmHg}, P=0.069)$. Resting HR between the two cohorts was similar (NT: $71.2 \pm 7.0 \mathrm{bpm}, \mathrm{AO}: 73.7 \pm 2.4 \mathrm{bpm}$, $P<0.05)$; however, the response to MINT exercise was significantly different $(P<0.01)$ with a reduction for the AO cohort (AO: $-2.6 \pm 1.9 \mathrm{bpm}$ ) and an increase for the NT cohort (NT: 4.8 $\pm 1.0 \mathrm{bpm}$ ) (Figure 3).

We previously reported that among the cohort of NT women in this research average acute $\mathrm{CV}$ responses may be driven by the mechanoreflex, as indicated by the significant correlations between grip performance and SBP ( $r=0.53, P<0.05)$, DBP $(r=0.55, P<0.05)$, and HR ( $r=0.65$, $P<0.01$ ) (Bentley and Thomas 2015, unpublished data). In comparison, the $\mathrm{CV}$ responses of $\mathrm{AO}$ women studied in the subsequent analysis of MINT are not correlated to either average RPE (SBP: 0.05, DBP: -0.1 , HR: 0.02, all $P>0.05$ ) or grip performance (SBP: 0.05, DBP: -0.06 , HR: -0.12 , all $P>0.05)$. The average acute responses of SBP, DBP, and HR were not correlated with any other variable, including state-trait anxiety, age, body mass index, resting SBP, or year since last menstrual period.

RPEs were similar between cohorts at both the midway (NT: 12.9 $\pm 2.6, \mathrm{AO}: 13.6 \pm 1.4)$ and end (NT: 13.5 \pm 2.9 , AO: $14.8 \pm 2.5)$ of $H G$ exercise with an average RPE (NT: 13.2 \pm 2.7 , AO: 14.2 $\pm 2.0, P=0.27$ ). Exit surveys completed by the NT group revealed that participants found the MINT protocol to be less enjoyable (MINT: 5.3 \pm 2.5 , ZONA: $8.03 \pm 1.4, P<0.05$ ), not as easy (MINT: $6.0 \pm 2.8$, ZONA: 


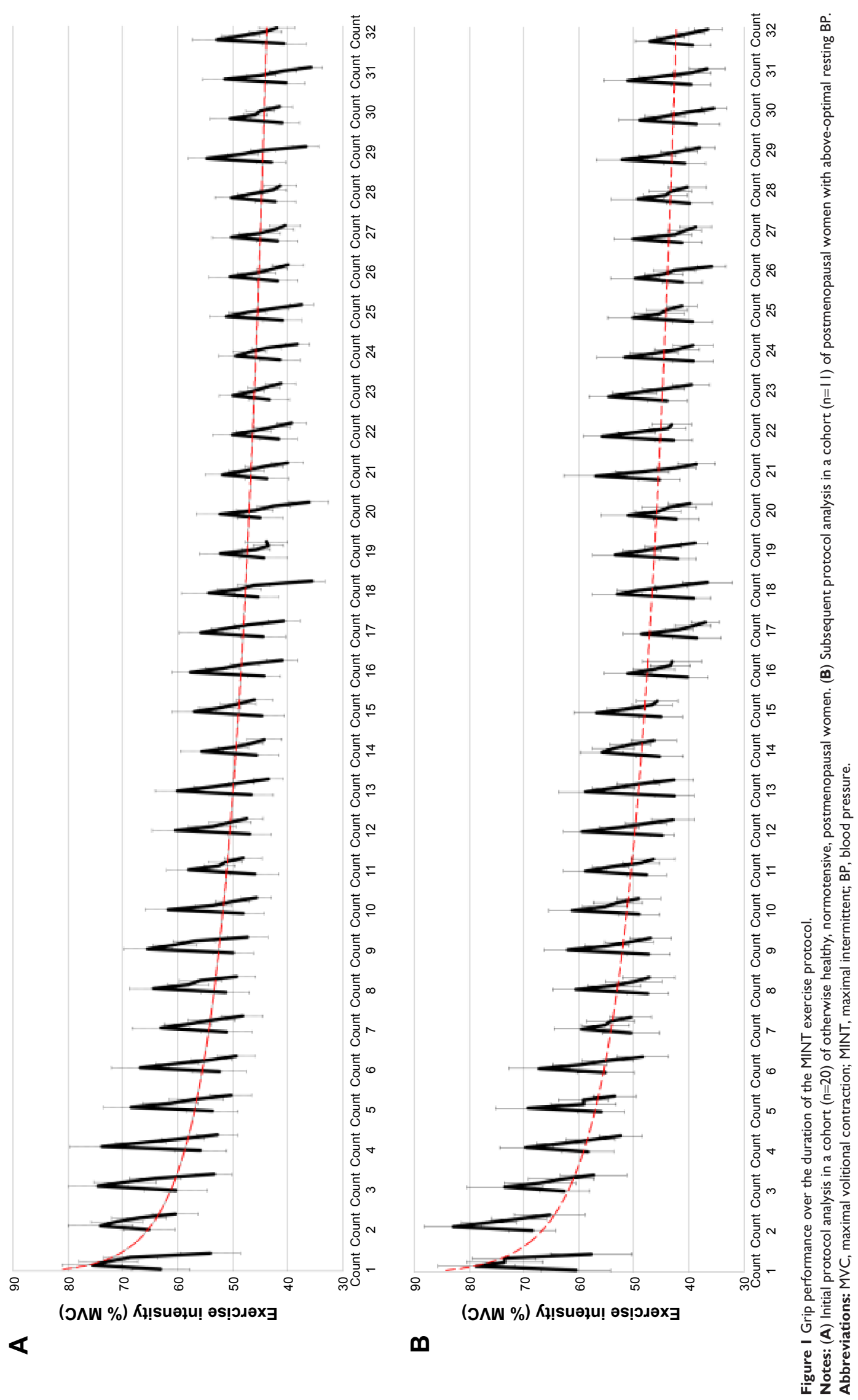




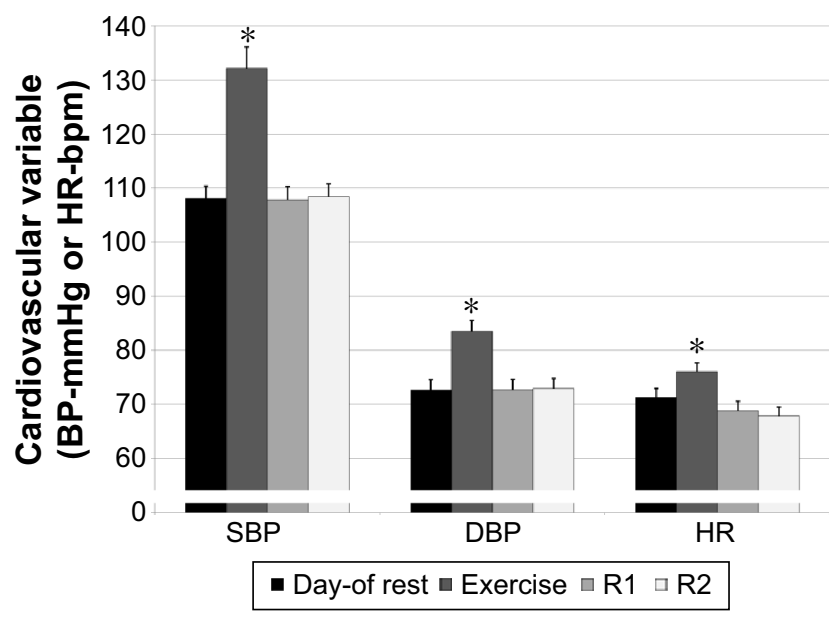

Figure 2 The acute cardiovascular responses of normotensive women to MINT. Note: As compared to day-of resting value, $* P<0.05$.

Abbreviations: MINT, maximal intermittent; RI, first recovery window (0-15 minutes); R2, second recovery window (I5-30 minutes); BP, blood pressure; $\mathrm{HR}$, heart rate; SBP, systolic blood pressure; DBP, diastolic blood pressure.

7.9 $\pm 2.0, P<0.01)$, with similar ratings of resulting fatigue (MINT: 5.1 \pm 2.7, ZONA: $4.3 \pm 2.8, P>0.05$ ). In line with these opinions, $25 \%$ of participants indicated that they would promote the MINT protocol to a friend and/or colleague versus $75 \%$ that indicated they would promote the ZONA protocol.
In the AO group, only one participant provided additional qualitative feedback for the MINT protocol directly saying that "it was easy" - participant LT14.

\section{Context}

As expected, grip performance throughout MINT was lower than the prescribed 100\% MVC. On average, participants exerted a grip force equivalent to approximately half of their 1-second average (NT: 50.4\%, AO: 49.7\%). Although the high-intensity MINT exercise protocol produced moderate acute changes to $\mathrm{BP}$, the resulting 5-second peak responses of SBP (NT: 150.3 $\pm 20.1 \mathrm{mmHg}, \mathrm{AO}: 175.2 \pm 20.6 \mathrm{mmHg}$ ) and DBP (NT: 89.6 $\pm 9.5 \mathrm{mmHg}, \mathrm{AO}: 99.4 \pm 20.1 \mathrm{mmHg}$ ) remained far below the thresholds of concern for exercise-induced BP response identified by the ACSM (SBP: $>250 \mathrm{mmHg}$, DBP: $>115 \mathrm{mmHg}) .{ }^{39}$

The AO women studied in the subsequent experimental analysis of MINT displayed no significant correlations of average $\mathrm{CV}$ reactivity to either RPE scores or performed grip intensity. Since neither indicators of central command nor indicators of mechanoreflex are linked to the $\mathrm{CV}$ response during MINT, it is reasonable to assume that perhaps the metaboreflex is driving the $\mathrm{BP}$ responses of $\mathrm{AO}$ women,

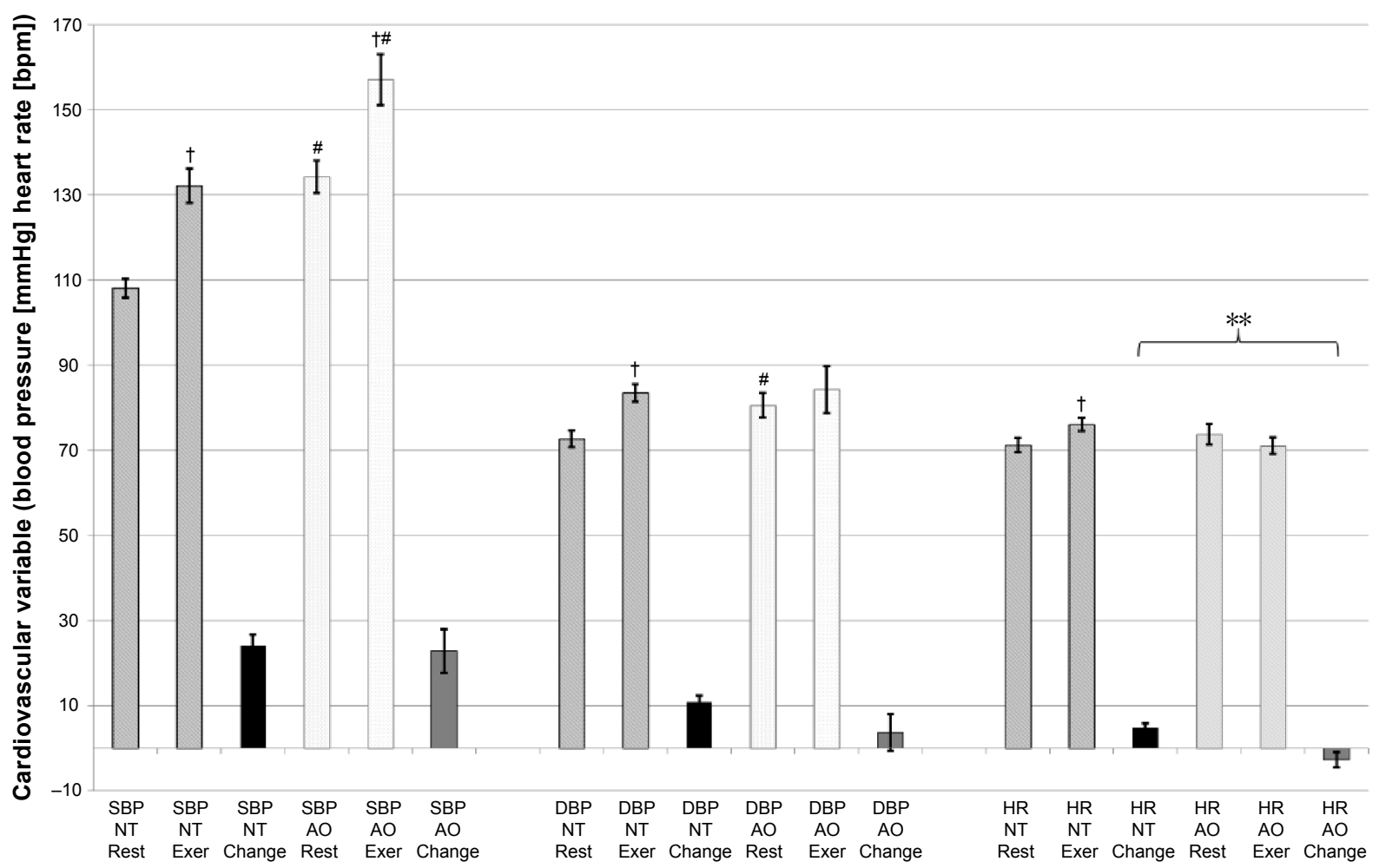

Figure 3 A direct comparison of the during-exercise cardiovascular responses to MINT for normotensive women (NT) and women with above-optimal resting blood pressures (AO).

Notes: Difference from resting ${ }^{t} P<0.05$, difference from $N T{ }^{*} P<0.05$, difference between change scores $* * P<0.01$.

Abbreviations: MINT, maximal intermittent; SBP, systolic blood pressure; DBP, diastolic blood pressure; HR, heart rate; Exer, exercise. 
despite efforts to reduce metabolite accumulation. In a study of older adults, enhanced metaboreflex sensitivity is reported among individuals with above-optimal resting BP with significantly stronger responses of SBP to postexercise ischemia (12 $\pm 3 \mathrm{mmHg}$ vs $6 \pm 1 \mathrm{mmHg}) .{ }^{40}$ Perhaps, the MINT protocol is effective in reducing, but not eliminating, local metabolite accumulation. Research with the primary goal of examining the ANS responses to HG exercise among these two groups of women is required to confirm current speculations. It would be extremely useful if such research also included older men for sex-specific comparisons of the ANS-driven pressor response to exercise.

Within the AO cohort, there were four women controlling their BP with either angiotensin-converting-enzyme inhibitors $(n=2)$, calcium channel blockers $(n=1)$, or diuretics $(n=1)$. An additional explorative analysis was conducted to determine the potential influence of medication use on the $\mathrm{BP}$ response to $\mathrm{HG}$ exercise. During exercise, those taking BP medication had an enhanced DBP response $(15.1 \pm 16.5 \mathrm{mmHg})$ compared to those not taking medication $(-2.8 \pm 8.7 \mathrm{mmHg})(P<0.05)$. There were no differences in the responses of SBP $(30.7 \pm 22.3 \mathrm{mmHg}$ vs $18.4 \pm 13.2 \mathrm{mmHg}$, $P>0.05)$ and HR $(+1.27 \pm 7.8 \mathrm{bpm}$ vs $-4.9 \pm 3.2 \mathrm{bpm}, P>0.05)$. Future research with adequate statistical power is required to adequately assess the impact of BP medication on the acute $\mathrm{CV}$ response to maximal $\mathrm{HG}$ exercise.

While participant feedback appears discouraging, further analysis of specific comments reveals that the physical discomfort of using the ADI instrument as the grip tool during the MINT protocol drove the negative reviews. "The texture of the devices is enormously different and influences one's response to both the device and the protocol" - participant ST09. "The ZONA protocol was frustrating to keep squeeze constant, but it was still more enjoyable than the cold steel, hard to hold, [ADI] tool" - participant ST15. Given that participants experienced only moderate RPEs and comparable levels of muscular fatigue between protocols, the maximal protocol design was accepted.

\section{Part 2: the MINT grip tool}

Participant feedback indicated that the in-laboratory grip tool (ADInstruments Inc.) was unpleasant and uncomfortable. This presented the opportunity to develop a grip tool that would be more acceptable for maximal HG exercise. The process of new product development (NPD) identifies activities commonly employed when creating and launching new products (Table 1). ${ }^{41}$ The precommercialization stages of NPD were followed in designing the MINT grip tool.

\section{Design and development stage} Research methodology

During the in-laboratory experimental analyses of the MINT exercise protocol, both NT women $(n=20)$ and AO women $(\mathrm{n}=11)$ used the ADI tool, a heavy $(287.7 \mathrm{~g})$ metal device with a rectangular shape (12.8 $\mathrm{cm}$ circumference). The NT women also completed a HG exercise bout on a separate research day using an alternative exercise tool, ZONA, which has a contoured shape ( $15.5 \mathrm{~cm}$ circumference) and weighs $162.7 \mathrm{~g}$ (Table 2). Feedback was collected from each group of women separately. As part of an exit survey, the NT cohort rated (1-10 scale) each tool (ADI and ZONA) for enjoyment, comfort, and provided net promoter scores (10-point

Table I Precommercialization stages of NPD with reference to the MINT grip tool development

\begin{tabular}{|c|c|c|}
\hline NPD stage name & Stage summary & Relationship to MINT grip tool \\
\hline New product strategy & $\begin{array}{l}\text { Links the NPD process to company objectives and } \\
\text { provides focus for idea/concept generation and } \\
\text { guidelines for establishing screening criteria. }\end{array}$ & $\begin{array}{l}\text { Product idea presented itself after initial feedback from research } \\
\text { participants. }\end{array}$ \\
\hline Idea generation & $\begin{array}{l}\text { Searches for product ideas that meet company } \\
\text { objectives. }\end{array}$ & \\
\hline Screening and evaluation & $\begin{array}{l}\text { An initial analysis to determine which ideas are } \\
\text { pertinent and merit more detailed study. }\end{array}$ & \\
\hline Business analysis & $\begin{array}{l}\text { Further evaluates the ideas on the basis of quantitative } \\
\text { factors, such as profits, return-on-investment, and } \\
\text { sales volume. }\end{array}$ & \\
\hline Design and development & $\begin{array}{l}\text { Turns an idea on paper into a product that is } \\
\text { demonstrable and producible. }\end{array}$ & Four mock-MINT tools were created. \\
\hline Testing & $\begin{array}{l}\text { Conducts commercial experiments necessary to verify } \\
\text { earlier business judgments. }\end{array}$ & $\begin{array}{l}\text { Mock-MINT tools were compared against the original two in- } \\
\text { laboratory tools using structured focus groups. The final output of } \\
\text { structured feedback acquisition was the resulting MINT grip tool. }\end{array}$ \\
\hline
\end{tabular}

Abbreviations: MINT, maximal intermittent; NPD, new product development. 
Table 2 Summary of grip tool design features for the new product development focus groups

\begin{tabular}{|c|c|}
\hline Handgrip tool & Features \\
\hline 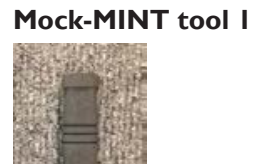 & $\begin{array}{l}\text { - Black color } \\
\text { - Rectangular (rounded edges) } \\
\text { - } 12 \mathrm{~cm} \text { central circumference } \\
\text { - "Supercycle foam handlebar grip" }\end{array}$ \\
\hline Mock-MINT tool 2 & $\begin{array}{l}\text { - Gray and black color } \\
\text { - Ergonomically contoured with } \\
\text { a round central core } \\
\text { - } 10.5 \mathrm{~cm} \text { inner, } 12 \mathrm{~cm} \text { central, } 12.5 \mathrm{~cm} \\
\text { outer circumferences } \\
\text { - Schwinn double-layer gel comfort } \\
\text { bike grips }\end{array}$ \\
\hline Mock-MINT tool 3 & $\begin{array}{l}\text { - Yellow color } \\
\text { - Rectangular } \\
\text { - } 10.5 \mathrm{~cm} \text { central circumference } \\
\text { - Wilson pro overgrip (tennis) }\end{array}$ \\
\hline Mock-MINT tool 4 & $\begin{array}{l}\text { - Pink color } \\
\text { - Rectangular } \\
\text { - I0.5 cm central circumference } \\
\text { - Tacki-Mac command hockey } \\
\text { grip slider }\end{array}$ \\
\hline ADI instrument & $\begin{array}{l}\text { - Gray color } \\
\text { - Rectangular } \\
\text { - I2.8 cm central circumference } \\
\text { - Metal }\end{array}$ \\
\hline ZONA & $\begin{array}{l}\text { - Gray and beige color } \\
\text { - Ergonomically contoured } \\
\text { - I5.5 cm central circumference } \\
\text { - Plastic }\end{array}$ \\
\hline
\end{tabular}

scale from 1 to 10). Participants also provided open-ended feedback regarding the grip tools. The AO cohort provided open-ended feedback regarding the ADI tool only at the end of the experimental visit.

\section{Results}

The NT women expressed greater enjoyment using the ZONA tool $(8.1 \pm 1.8)$ versus the ADI tool $(5.0 \pm 2.6)(P<0.01)$ and greater comfort using the ZONA tool $(9.0 \pm 0.9)$ versus the ADI tool $(4.4 \pm 2.6)(P<0.01)$. Only $35 \%$ of participants indicated that they would promote the ADI tool to a friend and/or colleague versus $90 \%$ that indicated they would promote the ZONA tool. A total of 14 women (70\%) chose to provide qualitative feedback on what would make an ideal HG tool, and this was combined with open-ended feedback from the eleven AO women. Thematic assessment revealed four primary areas: real-time feedback, grip material, physical shape, and circumference size. Two of the women expressed a desire for real-time digital feedback regarding grip force performance $(\mathrm{n}=1)$ and time remaining $(\mathrm{n}=1)$. Unfortunately, given that the primary goal of this research was to design a simple and accessible HG strategy, the decision was made not to incorporate a real-time feedback component. With regard to grip material, many thought that a softer $(n=8)$ grip with texture $(n=3)$ was desirable, with comfort $(n=3)$ and grip responsiveness $(n=2)$ as items to consider in tool creation. Finally, participants suggested that the tool has an ergonomic shape $(n=2)$ that is not too heavy $(n=1)$ with a small circumference $(n=3)$.

Using all aspects of participants' suggestions, four mockMINT grip tools were constructed from simple materials. The four mock-MINT grip tools that varied in size, shape, grip covering, and color progressed to the testing stage of product development.

\section{Testing stage Purpose}

To compare participant preferences and ratings of acceptability regarding four mock-MINT tool prototypes and two in-laboratory tools.

\section{Research methodology}

Postmenopausal women from the community were invited to participate in focus groups where they independently rated (using a 50-point scale from 0 [The Worst] to 10 [The Best]) the features of color, shape, size, and grip material of six separate tools. The characteristics of the tools (the ADI dynamometer, the ZONA dynamometer, and the four mockMINT tool prototypes) have been summarized in Table 2. Participants also used the net promoter scale to indicate how likely they would be to recommend each tool, with its current combination of features, to a friend. To conclude the focus group, participants independently selected their top choice of color, shape, size, and foam grip. Before leaving, the hand size of each participant was determined by measuring from the most distal tip of the third digit to the natural proximal wrist crease (approximately the distal edge of the radius). Participants' ratings of the grip tools were statistically compared using a 6 (tools) $\times 4$ (factors) two-way analysis of variance (ANOVA) with repeated measures, followed by multiple one-way ANOVAs with Bonferroni corrections as necessary. Pearson's correlations were completed 


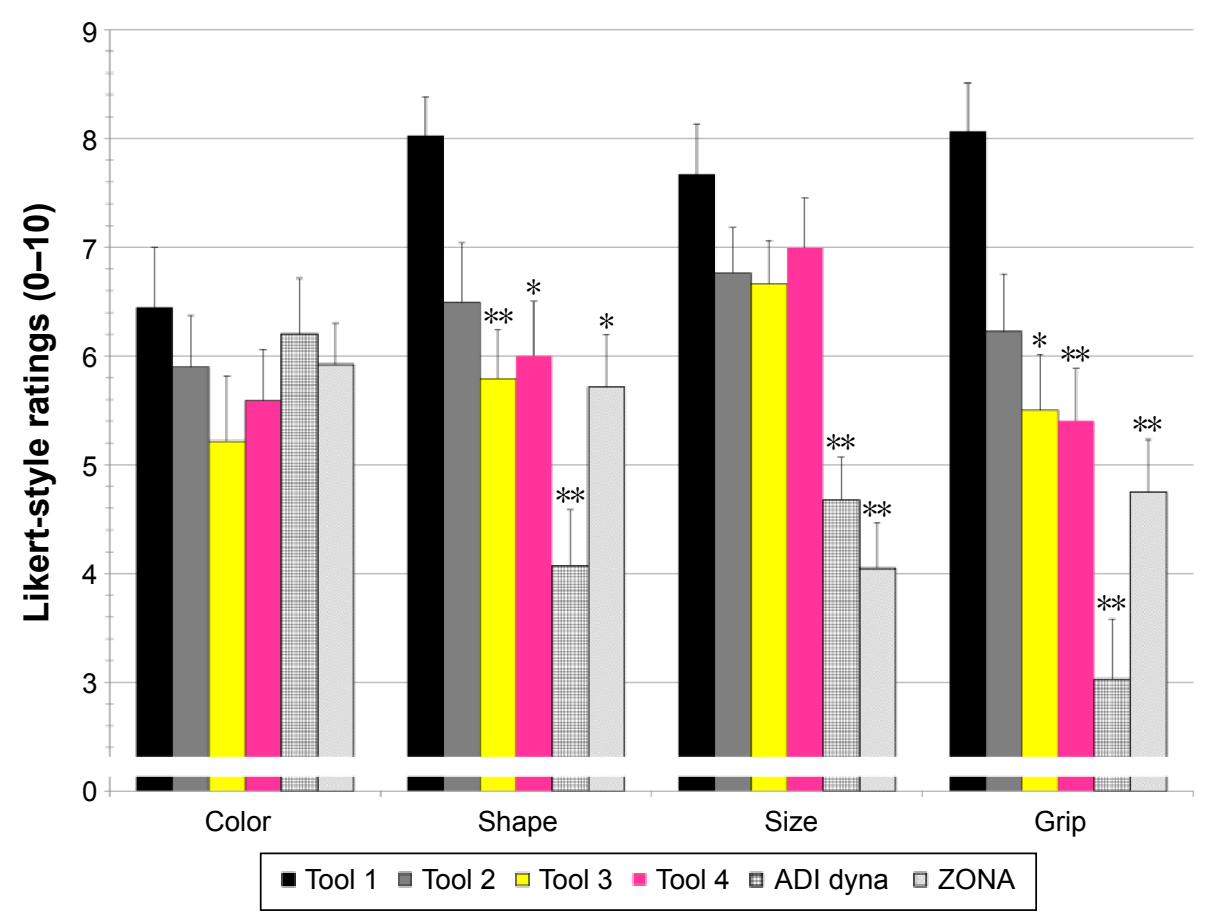

Figure 4 Feedback from the new product development focus groups. Notes: As compared to tool I, $* P<0.05, * * P<0.01$.

to assess relationships between factors. Significance was set at $P<0.05$.

\section{Results}

Twenty postmenopausal women from the community participated in focus groups. Participants were a combination of women who were new to the laboratory facilities $(n=9)$ and those who had previously completed other HG research studies $(\mathrm{n}=11)$. Two-way ANOVA revealed a significant interaction effect between tools and factors $(P<0.01)$ with corresponding post hoc comparisons revealing a robust preference for mock-MINT tool 1 for both shape and grip (Figure 4). When asked to select only one tool for each feature, mockMINT tool 1 was consistently selected the majority of the time (size: $55 \%$, shape: $50 \%$, color: $45 \%$, grip: $50 \%$ ), achieving an extremely high NPS ranking of $75 \%$. Mock-MINT tool 2 was the runner-up for all tool factors (size: $25 \%$, shape: $30 \%$, color: $25 \%$, grip: $40 \%$ ), with a corresponding NPS ranking of $50 \%$. Hand size was not correlated with tool size ratings for any of the tools ( $r$ range $=-0.03$ to 0.29 ). Interestingly, although the in-laboratory grip tools have specific features that make them desirable research tools, such as real-time grip force data (ADI instrument) and set training parameters (ZONA), their design features were rated extremely low with corresponding NPS ratings of $0 \%$ and $5 \%$, respectively.

\section{Context}

This research represents the testing stage of NPD, where six grip tools were rated by focus groups of postmenopausal women from the community. Although it was unanticipated that one tool would be favored across all tool dimensions, focus group participants preferred for all design features of the mock-MINT tool 1, including color, shape, size, and foam grip. As such, tool 1 with its current design features and high degree of population acceptability was selected as the MINT HG tool.

\section{Collective discussion}

This research describes the development and assessment of the MINT HG exercise strategy. The MINT HG exercise protocol was theoretically developed for enhanced accessibility by being easy to understand and simple to execute. MINT was first experimentally analyzed among a cohort of healthy postmenopausal women and was found to be both low risk (as determined by measuring both BP and HR reactions and postexercise CV recovery) and tolerable (as determined through participant opinions and measures of exercise performance). The MINT protocol was subsequently analyzed among a cohort of postmenopausal women with above-optimal BP and was confirmed to be low risk and tolerable. In addition to the MINT exercise protocol, this research also describes the development of a new product: the MINT 
grip tool. Research participants initially provided structured feedback regarding two in-laboratory devices that led to the design of four mock-MINT tool prototypes. Using focus groups, participant opinions were collected that informed the final MINT grip tool design. Together, the MINT exercise protocol and the MINT grip tool represent a highly accessible and participant-informed HG exercise strategy.

A strength of this research was the focused target population, postmenopausal women. As women age, their risk of CVD increases fourfold after the menopause transition. ${ }^{42}$ Controlling CVD risk factors, such as resting BP, can mitigate the impact of CVD as $>50 \%$ of all $\mathrm{CV}$ diseases are directly related to high $\mathrm{BP} .^{43} \mathrm{HG}$ exercise training has been used in the health literature as a strategy to effectively reduce BP with a surprising lack of research regarding potential sex-specific BP reductions. Furthermore, there is minimal research regarding the potentially disparate physiological responses of men and women to various HG exercise protocol design features (ie, intensity, duration of static contraction, work-to-rest ratios, etc). During sustained isometric HG contractions, women, compared to men, experience longer endurance times and less fatigue across a range of grip intensities from $30 \%$ to $75 \% \mathrm{MVC} .44,45$ In contrast, during intermittent (5-second contraction, 5-second relaxation) $\mathrm{HG}$ exercise at 50\% MVC intensity, this dichotomy in grip performance and fatigue disappears. ${ }^{32}$ The majority of previous research using $\mathrm{HG}$ exercise has recruited men, and this research is the first to design a HG exercise strategy specifically for postmenopausal women. Future experimental assessments of MINT should employ longitudinal designs to determine the potential training effect of MINT on resting BP. Such research would be especially impactful if proposed mediators of resting BP change were included as secondary outcome variables, such as improvements in autonomic nervous control of neurocardiac function.

\section{High-intensity grip safety}

Isometric exercise produces an acute pressure load on the heart by raising SBP and DBP spanning the duration of the exercise bout. The large BP responses have resulted in concerns regarding isometric exercise, especially in hypertensive populations. ${ }^{46,47}$ In response to these concerns, the American College of Sports Medicine recently published guidelines that identify concerning exercise BP values of $250 \mathrm{mmHg}$ systolic and $115 \mathrm{mmHg}$ diastolic. ${ }^{39}$ With these guidelines in mind, the current research used continuously collected BP and HR data to determine the $\mathrm{CV}$ response to high-intensity
HG exercise using a conservative approach of identifying 5 -second peak values. It was first determined that healthy normotensive women completing high-intensity intermittent $\mathrm{HG}$ exercise experienced peak BP responses below these published guidelines $(150 / 90 \mathrm{mmHg})$. High-intensity HG exercise was subsequently introduced to a cohort of women with above-optimal resting BP, confirming that $\mathrm{CV}$ responses during high-intensity HG exercise $(175 / 99 \mathrm{mmHg})$ remained below threshold values. Based on these results, future researchers should be confident in employing such a training program for at-home or unsupervised use among older women with resting BPs that extend into the classification of Stage 1 of hypertension.

\section{Health behavior}

The efficacy of health behavior change is often limited by low adherence and declining participation, despite the positive impact of incentive programs ${ }^{48}$ and robust intervention design for single health behavior change ${ }^{49}$ Uncharacteristically, researchers who used HG exercise report high exercise adherence to an individual exercise bout. ${ }^{33,34}$ One reason for this high adherence may be that $\mathrm{HG}$ exercise requires relatively little time as compared to traditional aerobic resistance training programs. The MINT protocol requires just over 5 minutes a day, approximately one-fifth the recommended daily aerobic duration. Similarly, strong $(>90 \%)$ adherence patterns are reported during $\mathrm{HG}$ training interventions for BP reduction lasting 4 weeks, ${ }^{50} 8$ weeks, ${ }^{34}$ and 10 weeks. ${ }^{51}$ However, a disadvantage of $\mathrm{HG}$ exercise is that we know very little about the additional health outcomes that commonly accompany traditional whole body programs (ie, improved blood lipid profile and alterations of body fat). Future research with the primary outcomes of assessing responses to HG exercise for such variables would be useful.

\section{Acknowledgments}

This study was supported in part by an internal Physical Education grant (Faculty of Kinesiology and Physical Education), University of Toronto. DCB is supported by a Canadian Institutes of Health Research CGS Doctoral award. The authors would like to thank Cindy Nguyen for her time computing real-time force outputs and Vanessa Dizonno for her time computing instantaneous cardiovascular responses. Finally, the authors would like to extend sincere thanks to all study participants for their time and dedication.

\section{Disclosure}

The authors report no conflicts of interest in this work. 


\section{References}

1. Crowe S, Fenon M, Hall M, Cowan K, Chalmer I. Patients', clinicians' and the research communities' priorities for treatment research: there is an important mismatch. Res Involv Engagem. 2015;1:2.

2. Chodzko-Zajko WJ, Proctor DN, Singh MAF, et al. Exercise and physical activity for older adults. Med Sci Sport Exerc. 2009;41(7): $1510-1530$.

3. Carlson DJ, Dieberg G, Hess NC, Millar PJ, Smart NA. Isometric exercise training for blood pressure management: a systematic review and meta-analysis. Mayo Clin Proc. 2014;89(3):327-334.

4. Lawrence MM, Cooley ID, Huet YM, Arthur ST, Howden R. Factors influencing isometric exercise training-induced reductions in resting blood pressure. Scand J Med Sci Sports. 2015;25(2):131-142.

5. Millar PJ, McGowan CL, Cornelissen VA, Araujo CG, Swaine IL. Evidence for the role of isometric exercise training in reducing blood pressure: potential mechanisms and future directions. Sports Med.2014; 44(3):345-356.

6. Inder JD, Carlson DJ, Dieberg G, McFarlane JR, Hess NC, Smart NA. Isometric exercise training for blood pressure management: a systematic review and meta-analysis to optimize benefit. Hypertens Res. 2016;39(2): 88-94.

7. Karinkanta S, Heinonen A, Sievänen H, Uusi-Rasi K, Fogelholm M, Kannus P. Maintenance of exercise-induced benefits in physical functioning and bone among elderly women. Osteoporos Int. 2009;20(4): 665-674.

8. Schenck-Gustafsson K. Risk factors for cardiovascular disease in women. Maturitas. 2009;63(3):186-190.

9. Kruger J, Carlson SA, Buchner D. How active are older Americans? Prev Chronic Dis. 2007;4(3):A53.

10. Bank AJ, Shammas RA, Mullen K, Chuang PP. Effects of short-term forearm exercise training on resistance vessel endothelial function in normal subjects and patients with heart failure. J Card Fail. 1998; 4(3):193-201.

11. Millar PJ, Bray SR, MacDonald MJ, McCartney N. The hypotensive effects of isometric handgrip training using an inexpensive spring handgrip training device. J Cardiopulm Rehabil Prev. 2008;28(3): 203-207.

12. Mostoufi-Moab S, Widmaier EJ, Cornett JA, Gray K, Sinoway LI. Forearm training reduces the exercise pressor reflex during ischemic rhythmic handgrip. J Appl Physiol. 1998;84(1):277-283.

13. Lin S, Chen Y, Li Y, Li J, Lu X. Physical ischaemia induced by isometric exercise facilitated collateral development in the remote ischaemic myocardium of humans. Clin Sci (Lond). 2014;127(10):581-588.

14. Kumar S, Seward J, Wilcox A, Torella F. Influence of muscle training on resting blood flow and forearm vessel diameter in patients with chronic renal failure. Br J Surg. 2010;97(6):835-838.

15. Piepoli M, Clark AL, Volterrani M, Adamopoulos S, Sleight P, Coats AJ. Contribution of muscle afferents to the hemodynamic, autonomic, and ventilatory responses to exercise in patients with chronic heart failure: effects of physical training. Circulation. 1996;93(5):940-952.

16. World Medical A. World Medical Association declaration of Helsinki: ethical principles for medical research involving human subjects. JAMA. 2013;310(20):2191-2194.

17. Liu S, Goodman J, Nolan R, Lacombe S, Thomas SG. Blood pressure responses to acute and chronic exercise are related in prehypertension. Med Sci Sports Exerc. 2012;44(9):1644-1652.

18. Ciriello J, Caverson MM, Polosa C. Function of the ventrolateral medulla in the control of the circulation. Brain Res. 1986;396(4):359-391.

19. Goodwin GM, McCloskey DI, Mitchell JH. Cardiovascular and respiratory responses to changes in central command during isometric exercise at constant muscle tension. J Physiol. 1972;226(1):173-190.

20. Krogh A, Lindhard J. The regulation of respiration and circulation during the initial stages of muscular work. J Physiol. 1913;47(1-2): $112-136$.

21. Williamson JW, McColl R, Mathews D, Mitchell JH, Raven PB, Morgan WP. Brain activation by central command during actual and imagined handgrip under hypnosis. J Appl Physiol. 2002;92(3): $1317-1324$
22. Kaufman MP, Hayes SG. The exercise pressor reflex. Clin Auton Res. 2002;12(6):429-439.

23. Hayward L, Wesselmann U, Rymer WZ. Effects of muscle fatigue on mechanically sensitive afferents of slow conduction velocity in the cat triceps surae. J Neurophysiol. 1991;65(2):360-370.

24. Kaufman MP, Longhurst JC, Rybicki KJ, Wallach JH, Mitchell JH. Effects of static muscular contraction on impulse activity of groups III and IV afferents in cats. J Appl Physiol Respir Environ Exerc Physiol. 1983; 55(1 Pt 1):105-112.

25. Mense S, Stahnke M. Responses in muscle afferent fibres of slow conduction velocity to contractions and ischaemia in the cat. JPhysiol. 1983;342:383-397.

26. Murphy MN, Mizuno M, Mitchell JH, Smith SA. Cardiovascular regulation by skeletal muscle reflexes in health and disease. Am J Physiol Heart Circ Physiol. 2011;301(4):H1191-H1204.

27. Barcroft $\mathrm{H}$, Millen JL. The blood flow through muscle during sustained contraction. J Physiol. 1939;97(1):17-31

28. Barnes WS. The relationship between maximum isometric strength and intramuscular circulatory occlusion. Ergonomics. 1980;23(4): 351-357.

29. Donald K, Lind A, Nicol G, Humphreys P, Taylor S, Staunton H. Cardiovascular responses to sustained (static) contractions. Circ Res. 1967;20(Suppl 1):15-30.

30. Choi HM, Stebbins CL, Nho H, Kim KA, Kim C, Kim JK. Skeletal muscle metaboreflex is enhanced in postmenopausal women. Eur J Appl Physiol. 2012;112(7):2671-2678.

31. Park SA, Kim JK. [Estrogen attenuates the pressor response mediated by the group III mechanoreflex]. J Korean Acad Nurs. 2011;41(2): 191-196. Korean.

32. Gonzales JU, Thompson BC, Thistlethwaite JR, Harper AJ, Scheuermann BW. Forearm blood flow follows work rate during submaximal dynamic forearm exercise independent of sex. $J$ Appl Physiol (1985). 2007;103(6):1950-1957.

33. Millar PJ, MacDonald MJ, McCartney N. Effects of isometric handgrip protocol on blood pressure and neurocardiac modulation. Int J Sports Med. 2011;32(3):174-180.

34. Badrov MB, Bartol CL, DiBartolomeo MA, Millar PJ, McNevin NH, McGowan CL. Effects of isometric handgrip training dose on resting blood pressure and resistance vessel endothelial function in normotensive women. Eur J Appl Physiol. 2013;113(8):2091-2100.

35. Jones H, George K, Edwards B, Atkinson G. Is the magnitude of acute post-exercise hypotension mediated by exercise intensity or total work done? Eur J Appl Physiol. 2007;102(1):33-40.

36. Spielberger C, RL G, RE L. Test Manual for the State Trait Anxiety Inventory. Palo Alto, CA: Consulting Psychologists Press; 1970.

37. Borg GA. Psychophysical bases of perceived exertion. Med Sci Sports Exerc. 1982;14(5):377-381.

38. Reichheld FF. The one number you need to grow. Harv Bus Rev, 2003; $81: 46-54$.

39. American College of Sports Medicine. ACSM's Guidelines for Exercise Testing and Prescription, Ninth Edition. Netherlands: Wolters Kluwer; 2013.

40. Sausen MT, Delaney EP, Stillabower ME, Farquhar WB. Enhanced metaboreflex sensitivity in hypertensive humans. Eur JAppl Physiol. 2009; 105(3):351-356.

41. Booz, Allen, Hamilton. New Product Management for the 1980's. New York, NY: Booz, Allen \& Hamilton, Inc.; 1982.

42. Yang XP, Reckelhoff JF. Estrogen, hormonal replacement therapy and cardiovascular disease. Curr Opin Nephrol Hypertens. 2011;20(2): 133-138.

43. World Health Organization. Cardiovascular Diseases, Fact sheet \#317. Vol. 2013. Geneva: WHO; 2013.

44. Petrofsky JS, Burse RL, Lind AR. Comparison of physiological responses of women and men to isometric exercise. J Appl Physiol. 1975;38(5): $863-868$. 
45. West W, Hicks A, Clements L, Dowling J. The relationship between voluntary electromyogram, endurance time and intensity of effort in isometric handgrip exercise. Eur J Appl Physiol Occup Physiol. 1995;71(4): 301-305.

46. Chrysant SG. Hemodynamic effects of isometric exercise in normotensive and hypertensive subjects. Angiology. 1978;29(5):379-385.

47. Mitchell JH, Wildenthal K. Static (isometric) exercise and the heart: physiological and clinical considerations. Annu Rev Med. 1974;25: 369-381.

48. Giles EL, Robalino S, McColl E, Sniehotta FF, Adams J. The effectiveness of financial incentives for health behaviour change: systematic review and meta-analysis. PLoS One. 2014;9(3):e90347.
49. Nigg CR, Long CR. A systematic review of single health behavior change interventions vs. multiple health behavior change interventions among older adults. Transl Behav Med. 2012;2(2):163-179.

50. Allen JD, Geaghan JP, Greenway F, Welsch MA. Time course of improved flow-mediated dilation after short-term exercise training. Med Sci Sports Exerc. 2003;35(5):847-853.

51. Badrov MB, Horton S, Millar PJ, McGowan CL. Cardiovascular stress reactivity tasks successfully predict the hypotensive response of isometric handgrip training in hypertensives. Psychophysiology. 2013;50(4): $407-414$.
Clinical Interventions in Aging

\section{Publish your work in this journal}

Clinical Interventions in Aging is an international, peer-reviewed journal focusing on evidence-based reports on the value or lack thereof of treatments intended to prevent or delay the onset of maladaptive correlates of aging in human beings. This journal is indexed on PubMed Central, MedLine,

\section{Dovepress}

CAS, Scopus and the Elsevier Bibliographic databases. The manuscript management system is completely online and includes a very quick and fair peer-review system, which is all easy to use. Visit http://www.dovepress. $\mathrm{com} /$ testimonials.php to read real quotes from published authors. 